\title{
The dielectric effect of xylene on an organoclay-containing composite
}

\author{
A. V. Shaw, S. T. H. Virtanen, A. S. Vaughan, T. Andritsch \\ The Tony Davis High Voltage Laboratory \\ University of Southampton \\ Southampton, UK \\ avb2g12@soton.ac.uk
}

\begin{abstract}
The influence of a non-polar solvent, xylene, on the electrical properties of a composite system containing polypropylene, copolymer ethylene/(vinyl acetate) and an organoclay has been considered since the synthesis of composites often involves a solvent. The presence of the organoclay has been shown to increase absorption of xylene; elsewhere similar effects have been associated with segregation of mobile species to nanofiller interfaces. Although the presence of neither the organoclay nor the xylene, in isolation, has a marked effect on the dielectric response of the system, when present together strong synergistic effects are seen, which we interpret in terms of solvent mediated relaxation of amphiphilic compatibilizer moieties introduced into the organoclay to aid processability. These local relaxation processes appear adversely to affect the breakdown strength of the system.
\end{abstract}

Keywords - Polypropylene, organoclay, xylene, composites

\section{INTRODUCTION}

The electrical properties of nanocomposite materials has been an active areas of research for almost three decades [1]. While numerous reports exist of improved properties on addition of a nanofiller, many others indicate that inclusion of nanoparticles can have negative consequences. While the precise origins of this irreproducibility are uncertain, what is clear is that small changes in processing or composition can exert a major effect on macroscopic electrical properties. [2]

It is well known that the presence of water can alter the dielectric properties of nanocomposites. For example, Ayoob et al. [3] found that the permittivity increased with nanofiller content when samples were immersed in water, while Lau et al. found there to be a significant difference in the dielectric response of polyethylene after immersion in water, depending on the surface treatment of the nanofiller [4]. These effects were interpreted in relation to the adsorption of dipolar water molecules at nanoparticle surfaces, whereupon, the precise dynamics were governed by local hydrogen bonding effects.

While absorption of water will be an inevitable consequence of environmental exposure, this is not the only labile molecular species that may be present, since a solvent processing step is often used in the production of nanocomposites. The work described here therefore set out to examine the influence of a labile, but non-polar, species on the electrical characteristics of a polymeric nanocomposite.

In this study, a polymer blend system based on isotactic polypropylene (iPP) and ethylene/(vinyl acetate) (EVA) copolymer were employed, where the role of the latter was to act to increase compatibility with an organically functionalized nanoclay. Specifically, the influence of retained solvent, xylene, on the dielectric response of the system was examined.

\section{EXPERIMENTAL}

\section{A. Sample Synthesis and Conditioning}

The following materials were used: iPP, product number 427888 from Sigma Alrich, and EVA, Elvax 750 containing 9 wt.\% VA, from DuPont. The organoclay was used as obtained from Sigma Aldrich (product number: 682624). All of the samples used here contained, by weight, 80 parts of iPP to 20 parts of EVA and will be referred to by their respective nominal organoclay loading: $0,2.5$ or 5 wt. \%.

The material preparation procedure used here was adapted from published studies [5], [6]. The organoclay was first sonicated in xylene for $30 \mathrm{~min}$ before being added, along with the EVA, into a rotary evaporator at $140{ }^{\circ} \mathrm{C}$. When the polymer had completely dissolved, the iPP was added and the temperature was increased to $155{ }^{\circ} \mathrm{C}$ to aid dissolution of the iPP. The mixture was left stirring for $30 \mathrm{~min}$ before the solvent was removed under vacuum. From the resulting product, samples of required thickness $(100 \mu \mathrm{m})$ were melt-pressed using a hydraulic press. After being held in the melt phase for 5 min, the films were crystallized isothermally at $120{ }^{\circ} \mathrm{C}$ for 10 min, before being quenched into a water bath at room temperature. The required temperature and time were determined using previous analysis using differential scanning calorimetry.

All of the films were then further dried for one week under dynamic vacuum at $70{ }^{\circ} \mathrm{C}$, whilst monitoring the mass change, to remove any absorbed water or residual solvent. These samples will hereafter be referred to as dried. Subsequent immersion in xylene, for 21 days, was then used to introduce this solvent and, again, the mass was monitored throughout. These samples will hereafter be referred to as immersed.

\section{B. Sample Analysis}

Thermogravimetric analysis (TGA) was performed in air up to $800{ }^{\circ} \mathrm{C}$ at a heating rate of $10^{\circ} \mathrm{C}$ per minute using a Perkin Elmer Pyris 1 instrument. Fourier-transform infrared (FT-IR) spectra were acquired using a Thermo Scientific Nicolet iS5 operating in attenuated total internal reflectance (ATR) mode.

AC breakdown strength was measured in accordance with ASTM D149-97a. For this, the sample was submerged in silicone oil between vertically opposed spherical electrodes (6.3 mm diameter). Each system was tested twenty times using a ramp rate of $500 \mathrm{~V} / \mathrm{s}$ and the results were analyzed using twoparameter Weibull statistics with $90 \%$ confidence limits, within the Origin software package.

Dielectric spectra were acquired using a Solartron 1296 dielectric interface together with a Schlumberger SI1260 impedance/phase gain analyzer system. For this, an AC voltage of amplitude 1V was applied across electrodes $30 \mathrm{~mm}$ in 
diameter with a guard electrode; the frequency range of $10^{-1}$ to $10^{5} \mathrm{~Hz}$ was considered.

\section{RESULTS AND DISCUSSION}

Fig. 1 shows TGA data obtained from both dried and immersed samples. Consider first the results obtained from the dried samples. The absence of any detectable changes in mass at low temperatures indicates that negligible volatile species remained entrained within the systems after drying and that the actual organoclay loading level present in each of the filled systems were 2.1 and 5.1 wt. \% for the composition goals of 2.5 and 5 wt. \% respectively. For simplicity, the paper will, nevertheless, still refer the samples by their nominal target composition. The inset in Fig. 1 shows low temperature TGA data obtained from samples that had been immersed in xylene. Here, significant mass loss is evident in all three traces, which we attribute to the loss of absorbed xylene. Furthermore, in these samples, the observed mass loss increases with organoclay loading, indicating that the organoclay plays a significant role in the material susceptibility to xylene absorption.

Fig. 2 shows FT-IR data acquired from films of each material after one week drying in a vacuum oven. In the materials containing organoclay there is an additional broad peak around $1075 \mathrm{~cm}^{-1}$, corresponding to the $\mathrm{Si}-\mathrm{O}$ bonding in the clay. However, no clear differences between spectra obtained before and after drying are evident. It is particularly notable that no absorption peaks can be seen in spectral regions that would correspond to degradation of the polymer during the extended drying procedure. This confirms that the drying procedure did not chemically alter the films. Repeating the FTIR analysis after immersion in xylene provided no clear indication of additional vibrational modes associated with the xylene aromatic ring. In view of the TGA results presented above, this is surprising since the concentration should be well within the general detection limits of the technique. We therefore suggest that this result is a consequence of the adoption here of the ATR approach, which is highly surface specific. That is, the local (surface) concentration of xylene is rather different from that present in the bulk.

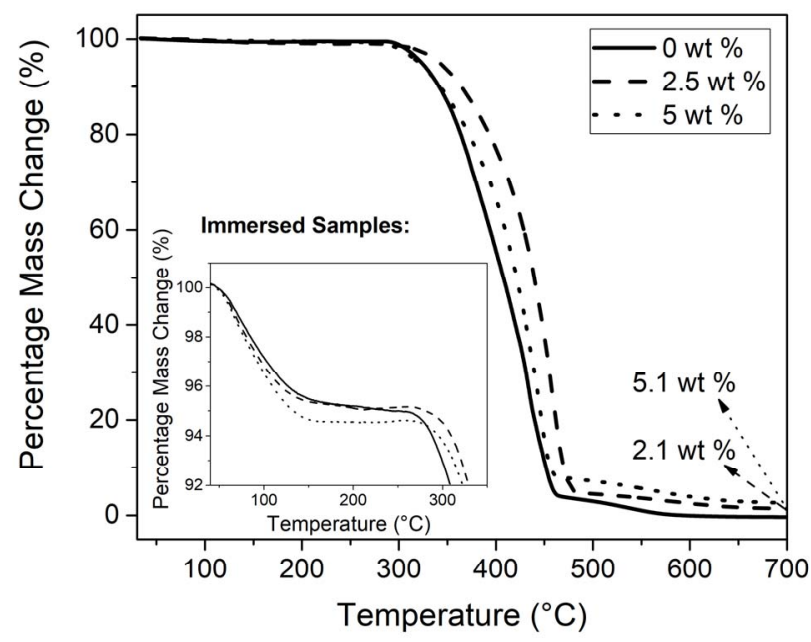

Fig. 1. Data from TGA conducted in air on the dried samples and the immersed samples (inset).
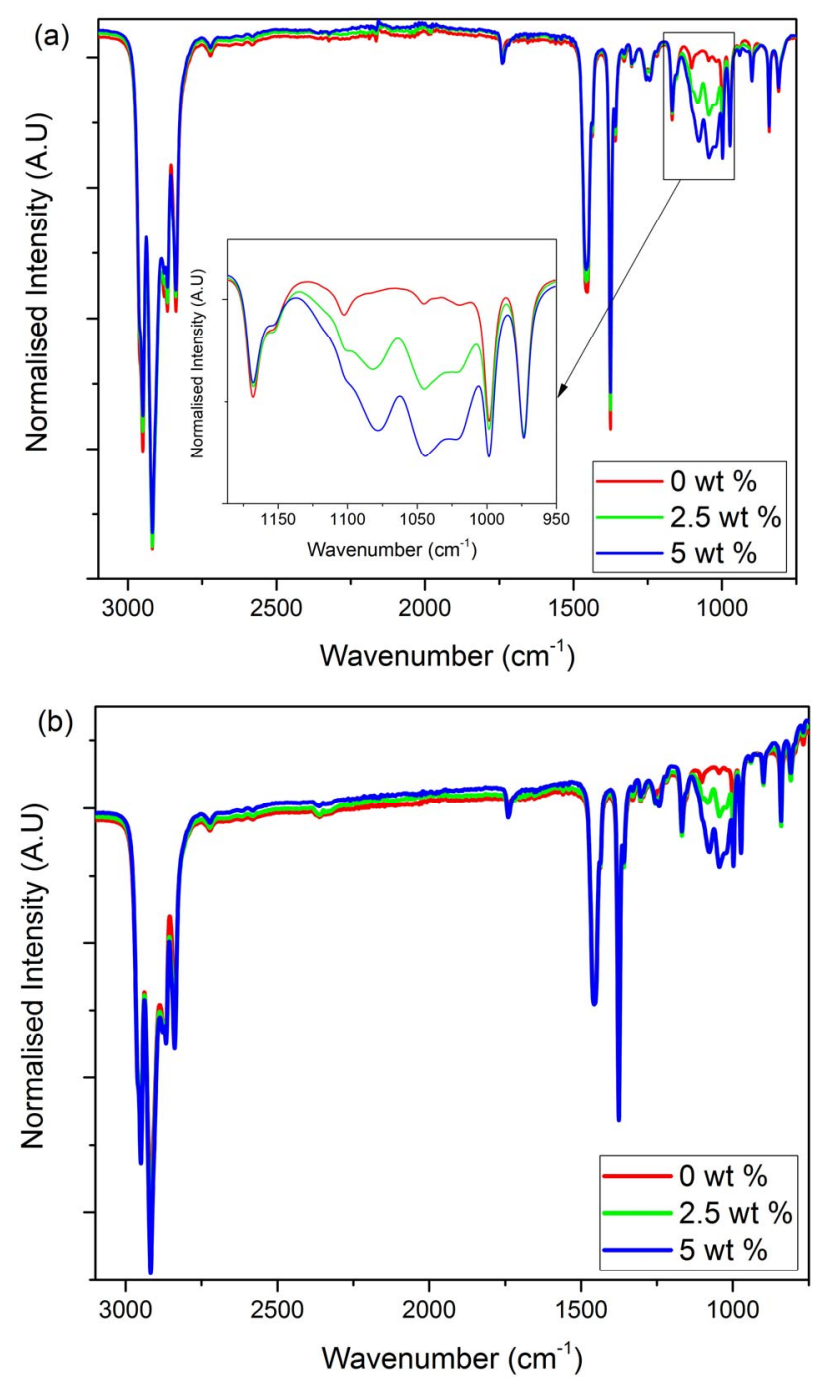

Fig. 2. FT-IR data, normalized using Z-scores, of the samples showing (a) data from the dried samples showing no evidence of polymer degradation (for example, oxidation) and the organoclay loading (inset) and (b) data from the immersed samples showing no evidence of xylene.

Fig. 3 shows dielectric spectroscopy data from all three sample compositions after different immersion times in xylene. For the samples without organoclay, although some apparent variations in real relative permittivity, $\varepsilon^{\prime}$, can be seen, these are well within experimental uncertainties and are associated with errors/variations in the actual sample thickness. Indeed, the value predicted by the Lichtenecker Rother equation [7] for the real relative permittivity of an 80:20 blend of iPP and EVA is 2.4. The imaginary part of the relative permittivity, $\varepsilon^{\prime \prime}$, is also found to be independent of immersion time/xylene content, which indicates that the presence of small quantities of this non-polar species has negligible effect on the dielectric response of the system, a conclusion that is entirely in line with expectations.

However, in the case of the samples containing the organoclay, the effect of xylene immersion time is very different. Here, a pronounced increase in $\varepsilon^{\prime}$ is evident with decreasing frequency which, from the imaginary data, appear to be associated with two processes, a distinct dielectric loss process around $10-10^{3} \mathrm{~Hz}$, plus a low frequency dispersion which may either be related to Maxwell Wagner polarization or 


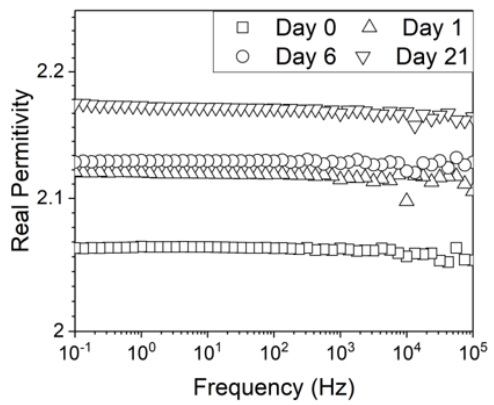

d)

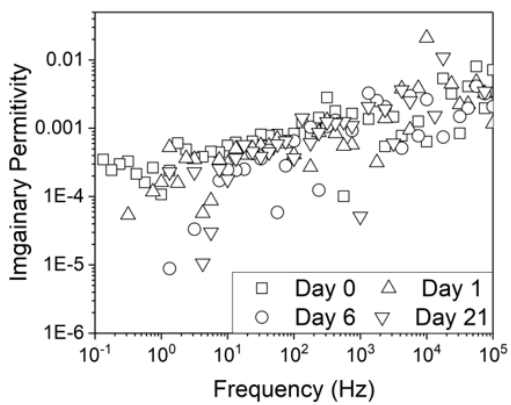

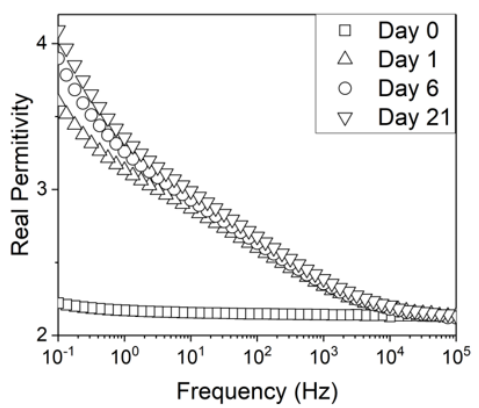

e)

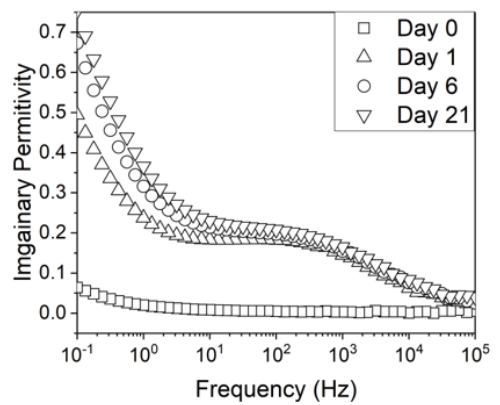

c)

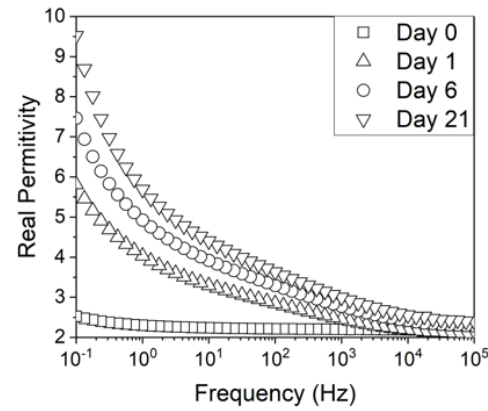

f)

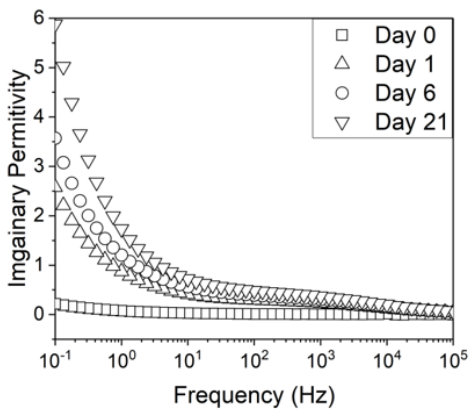

Fig. 3. Real relative permittivity of the composites containing (a) 0 wt. \%, (b) 2.5 wt. \% and (c) 5 wt. \% organoclay and the imaginary permittivity of the composites containing (d) 0 wt. \%, (e) 2.5 wt. \% and (f) 5 wt. \% organoclay after different time intervals of immersion in xylene.

to increasing DC conduction. Evidently, the strength of these loss processes increases with both immersion time and organoclay loading.

We believe these observations are a result of the organoclay-xylene interactions. In general, organoclays contain amphiphilic molecules that are introduced within the galleries, both to increase compatibility with the host matrix and to increase the interlayer spacing and, thereby, ease exfoliation. In the absence of xylene, the presence of the organoclay itself has little effect on the overall dielectric response of the system. However, addition of xylene - which also, in isolation, does not contribute to the permittivity - then leads to the dramatic changes in the dielectric response shown in Fig. 3. We suggest that these changes result from synergistic interactions between the non-polar solvent molecules and components present within the clay, the most obvious candidate molecular species being the amphiphilic compatibilizer. Specifically, we propose that the presence of the xylene solvent facilitates dipolar species to respond to the applied field, such that the above effects are facilitated.

If the xylene were to remove the organic chains from the organoclay, this would cause a change in the composition of the system and, therefore, its properties. Since the penetration of the xylene through the polymer matrix has been confirmed by TGA and inferred from dielectric spectroscopy, it was considered necessary to investigate if xylene could affect the organoclay in an irreversible manner. To do this, pristine organoclay was soaked in xylene for a week before taking FTIR spectra of the xylene, since it was considered that due to the low loading of the organoclay in the soaked samples and the relatively large volume of xylene that the samples had been soaked in, any organic species extracted in the process would not be detectable due to their low concentration. The spectra obtained from the xylene that had been soaking the pristine organoclay showed no difference to the pristine xylene, suggesting that the xylene does not extract any components from the organoclay.

The above dielectric results have some parallels with previous studies of water absorption effect in nanocomposites. Lau et al. [4] reported that samples immersed in water exhibited a dielectric response that was strongly influenced by local effects; that is, the dynamics of the dipole (in this case water) were constrained by the hydrogen bonding on the nanosilica filler employed. We consequently suggest that the dipoles that lead to the effects shown above in Fig. 3 originate in the organoclay and are able to respond to the applied field due to the presence of xylene, which effectively solvates them, promoting their mobility. Hosier et al. [8] noted that, although there are relatively few studies in the literature on the effect of water on breakdown, such work consistently shows that the presence of water adversely affects breakdown strength. Since it would appear that xylene-mediated dielectric effects parallel those seen when water is present, it is appropriate to consider how the relaxation processes evident in Fig. 3 influence breakdown performance.

AC dielectric breakdown test data are summarized in Fig. 4, where the error bars correspond to the $90 \%$ confidence intervals obtained from two parameter Weibull analysis. In the case of the unfilled samples, where the presence of the absorbed xylene does not affect the dielectric relaxation, the breakdown strength of the dried and immersed samples is equivalent, within experimental uncertainties. In the systems containing the organoclay, the presence of the xylene, which in the unfilled system is benign, leads to a significant reduction in 


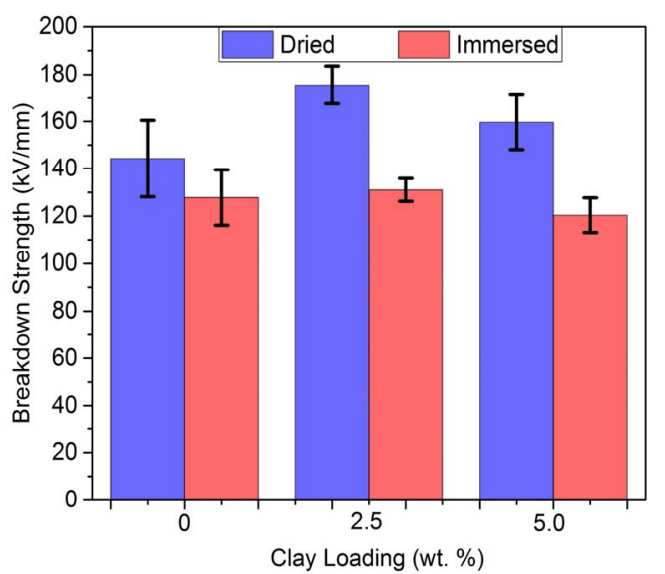

Fig. 4. Summary plot of the alpha values obtained from two parameter Weibull statistics of the AC dielectric breakdown data together with their associated errors.

breakdown strength. Therefore, it would appear that the presence of xylene promotes a mechanism that causes breakdown to occur at a reduced electric field; this is, phenomenologically, similar to work described elsewhere, which reported on the effects of absorbed water on breakdown strength.[8] In the case of water, it is possible to propose a number of mechanisms by which its presence could adversely affect breakdown strength, involving dipolar and/or ionic processes; here, the critical role played by the nonpolar xylene combined with the mediating effect of this species in increasing dielectric losses at $50 \mathrm{~Hz}$ (the frequency of the dielectric breakdown testing), suggests that the former is most pertinent.

\section{CONCLUSIONS}

The influence of a labile, nonpolar molecule on dielectric response and breakdown behavior has been examined in a polymer blend system containing varying loading levels of an organoclay. In the absence of xylene, addition of the organoclay has little effect on the dielectric response of the system and, at low loading levels, appears to enhance the breakdown strength of the matrix. Equally, in the absence of the organoclay, the presence of xylene has an insignificant effect on both dielectric response and breakdown. However, when both the organoclay and the xylene are present together, strong synergistic effects are seen. Specifically, here, dielectric losses are markedly increased and, in parallel, AC breakdown strength is reduced. We propose that these effects are associated with solvation of amphiphilic species that are added to the virgin clay in order to render it more compatible with the host polymer, thereby easing dispersion during processing.

While it is generally accepted that in nanocomposite systems, optimized properties require good dispersion of the nanoclay, the work presented here, we suggest, indicates that where dielectric and electrical properties are concerned, improved dispersion is not the panacea it may be elsewhere. Rather, the mechanisms that determine bulk properties can be influenced by local physical and chemical factors. While it is conceptually easy to envisage how absorbed water can couple with an applied electric field and, thereby, affect dielectric response, it is less obvious that the presence of a nonpolar species can serve to facilitate comparable effects. Here, the presence of the nonpolar xylene leads, indirectly, to phenomena that mirror the direct effects of absorbed water. While both are, practically, deleterious, this does not eliminate the possibility of other labile species acting in some advantageous way, by which macroscopic electrical properties are enhanced.

\section{ACKNOWLEDGMENT}

The authors would like to acknowledge the support of AWE plc, EPSRC and the support of Dr Matthew A. Brown.

All data supporting this study are openly available from the University of Southampton repository at http://dx.doi.org/10.5258/SOTON/D0518

\section{REFERENCES}

[1] T. J. Lewis, "Interfaces: Nanometric dielectrics," IEEE Trans. Dielectr. Electr. Insul., vol. 1, no. 5, pp. 202-212, 1994.

[2] M. Bhattacharya, "Polymer nanocomposites-A comparison between carbon nanotubes, graphene, and clay as nanofillers," Materials (Basel)., vol. 9, no. 4, pp. 1-35, 2016.

[3] R. Ayoob, T. Andritsch, and A. S. Vaughan, "Water absorption behaviour in polyethylene boron nitride nanocomposites," Proc. 2016 IEEE Int. Conf. Dielectr. ICD 2016, vol. 2, pp. 784-787, 2016.

[4] K. Y. Lau, A. S. Vaughan, G. Chen, I. L. Hosier, and a F. Holt, "On the dielectric response of silica-based polyethylene nanocomposites," J. Phys. D. Appl. Phys., vol. 46, no. 9, 2013.

[5] I. L. Hosier, A. S. Vaughan, and S. G. Swingler, "Structure property relationships in polyethylene blends : the effect of morphology on electrical breakdown strength," J. Mater. Sci., vol. 2, pp. 4523-4531, 1997.

[6] K. Y. Lau, A. S. Vaughan, G. Chen, and I. L. Hosier, "Polyethylene nanodielectrics: The effect of nanosilica and its surface treatment on electrical breakdown strength," Annu. Rep. - Conf. Electr. Insul. Dielectr. Phenomena, CEIDP, pp. 21-24, 2012.

[7] J. A. Reynolds and J. M. Hough, "Formulae for dielectric constant of mixtures," Proc. Phys. Soc. Sect. B, vol. 70, no. 8, pp. 769-775, 1957.

[8] I. Hosier, M. Praeger, A. Holt, A. Vaughan, and S. Swingler, "On the Effect of Functionaliser Chain Length and Water Content in Polyethylene/Silica Nanocomposites: Part I Dielectric Properties and Breakdown Strength," IEEE Trans. Dielectr. Electr. Insul., vol. 24, no. 3, pp. 1698-1707, 2017. 